\title{
Exact Relativistic Magnetized Haloes around Rotating Disks
}

\author{
Antonio C. Gutiérrez-Piñeres ${ }^{1,2}$ and Abraão J. S. Capistrano ${ }^{3,4}$ \\ ${ }^{1}$ Facultad de Ciencias Básicas, Universidad Tecnológica de Bolívar, CP 131001, Cartagena, Colombia \\ ${ }^{2}$ Instituto de Ciencias Nucleares, Universidad Nacional Autónoma de México, AP 70543, 04510 México, DF, Mexico \\ ${ }^{3}$ Federal University of Latin-American Integration, P.O. Box 2123, 85867-670 Foz do Iguaçu, PR, Brazil \\ ${ }^{4}$ Casimiro Montenegro Filho Astronomy Center, Itaipu Technological Park, 85867-900 Foz do Iguaçu, PR, Brazil
}

Correspondence should be addressed to Antonio C. Gutiérrez-Piñeres; gutierrezpac@gmail.com

Received 16 August 2015; Revised 9 September 2015; Accepted 18 November 2015

Academic Editor: Klaus Kirsten

Copyright ( 2015 A. C. Gutiérrez-Piñeres and A. J. S. Capistrano. This is an open access article distributed under the Creative Commons Attribution License, which permits unrestricted use, distribution, and reproduction in any medium, provided the original work is properly cited.

\begin{abstract}
The study of the dynamics of magnetic fields in galaxies is one of important problems in formation and evolution of galaxies. In this paper, we present the exact relativistic treatment of a rotating disk surrounded by a magnetized material halo. The features of the halo and disk are described by the distributional energy-momentum tensor of a general fluid in canonical form. All the relevant quantities and the metric and electromagnetic potentials are exactly determined by an arbitrary harmonic function only. For instance, the generalized Kuzmin-disk potential is used. The particular class of solutions obtained is asymptotically flat and satisfies all the energy conditions. Moreover, the motion of a charged particle on the halo is described. As far as we know, this is the first relativistic model describing analytically the magnetized halo of a rotating disk.
\end{abstract}

\section{Introductory Remarks}

In the observational context, many ambiguities still exist about the main constituents, geometry, and dynamics (thermodynamics) of the galactic disk-haloes. However, there are several different observations which probe the galactic and surrounding galactic magnetic field. For instance, it can be measured by nonthermal radio emission from energy equipartition that results from the interaction of magnetic energy with relativistic particles which can play a role in the formation of arms in spiral galaxies (see Krause's "Magnetic Fields and Halos in Spiral Galaxies" [1] and "Magnetic Fields in Spiral Galaxies" [2] and references therein). For nearby galaxies, other probes are used as optical polarization, polarized emission of clouds and dust grains, maser emissions, diffuse radio polarized emission, and rotation measures of background polarization sources also. Magnetic fields can be seen as a new structural quantity and added to other constituent parameters (e.g., $H$-alpha lines, density mass, local shocks, etc.) are important to study the formation and dynamics of galaxies. Other works propose that magnetic fields also play important role in large-structure formation [3].
It is important to stress that magnetic fields are found mainly in interstellar medium and can be found in every type of galaxies but remarkably noticed in spiral galaxies (see [4] and references therein). For instance, Milky Way has been actively studied in its three regions (central bulge, halo, and accretion disk). This motivates obtaining a proper description of magnetic fields from an oriented general principle and relativistic thin disks have been revealed as one of the best ways to study them. Relativistic disks were also extensively studied in literature in several configurations. Exact solutions that have relativistic static thin disks as their sources were first studied by Bonnor and Sackfield [5] and T. Morgan and L. Morgan [6, 7]. Subsequently, several classes of exact solutions corresponding to static [8-17] and stationary [18-22] thin disks have been obtained by different authors.

The superposition of a static or stationary thin disk with a black hole has been considered in [23-31]. Thin disks around static black holes in a magnetic field have been studied in [32]. Relativistic disks embedded in an expanding FriedmannLemaître-Robertson-Walker universe have been studied in [33] and perfect fluid disks with halos in [34]. Furthermore, the stability of thin disks models has been investigated using a first-order perturbation of the energy-momentum tensor 
in [35]. On the other hand, thin disks have been discussed as sources for Kerr-Newman fields [36, 37], magnetostatic axisymmetric fields [38, 39], and conformastatic and conformastationary metrics [40-42]. Also, models of electrovacuum static counterrotating dust disks were presented in [43], charged perfect fluid disks were studied in [44], and charged perfect fluid disks were presented as sources of static and Taub-NUT-type spacetimes in $[45,46]$. Also, monopole and dipole layers in curved spacetimes were analyzed in [47] and electromagnetic sources distributed on shells in a Schwarzschild background in [48].

The thin disks with magnetic fields studied in [3638] were obtained by means of the well-known "displace, cut, and reflect" method. Solutions that can be interpreted as thin disks of finite extension can be obtained if an appropriate coordinate system is introduced. A coordinate system that adapts naturally to a finite source and presents the required discontinuous behavior is given by the spheroidal coordinates. Some examples of finite thin disks obtained from vacuum solutions expressed in these coordinates can be found in $[5,6,9,11]$ and from electrovacuum solutions in [42].

Moreover, interestingly, magnetic fields seem to play an important role in the formation of jets (resulting from collimated bipolar outflows of relativistic particles) and the accretion disk near supermassive black holes [49]. A current revision of the status of our knowledge about the magnetic fields in our Milky Way and in nearby star-forming galaxies is summarized in [50]. Additionally, a study of the disk and halo rotation is reported in [51], whereas the possibility of magnetic fields that can be generated in the outskirts of disks is studied in [52]. Solutions for the Einstein and EinsteinMaxwell field equations which are consistently applicable to the context of astrophysics remain a topical problem. Nevertheless, the effects of magnetic fields on the physical processes in galaxies and their disk-halo interaction have been scarcely considered in the past. Similarly, the relevance of relativistic models of disks around black holes in a magnetic field is discussed in [32].

The presence of the electric field on the dark matter halo models has been considered in [53], whereas the presence of electromagnetic field in the halo-disk system has been studied in $[54,55]$ in which the gravitational sources are statics. In [54] we provided detailed overview of the research in the relativistic disks; accordingly we will not repeat them here.

In this paper, we do not focus on the whys and wherefores of the detailed discussion on the probes and relevance of magnetic fields on disks but we apply a standard galaxy modeling as a stationary thin disk and, correspondingly, we associate the halo with the region surrounding the disk. We present the conformastationary version of the static thin disk-halo systems studied in [54]. In addition, we study the features of the principal quantities characterizing the dynamic of the magnetized haloes corresponding to the disks presented in [56]. Therefore, we take the definition in [57] as standard, following the original terminology by Synge [58]: conformastationary are those stationary spacetimes with a conformally flat space of orbits.

Accordingly, we show that the rotating disk-haloes with isotropic pressure, stress tensor, and heat flow generalize the static disk-haloes obtained in [54]. Our results are compatible with those presented in [53] on possible features of galactic halo. Moreover, the description of the motion of charged particles on disk is deduced and is in agreement with the results of the similar analysis discussed in [59]. As far as we know, this is the first relativistic model describing analytically the relativistic magnetized halo of a rotating disk.

The paper is organized as follows. In Section 2, the distributional Einstein-Maxwell equations for haloes surrounding thin disks are obtained. In Section 3 we obtain expressions, in terms of an arbitrary harmonic function, for the most important quantities characterizing the dynamic of the disk and halo. In Section 4 we first calculate quantities for harmonic function described by the generalized Kuzmin-disk potential. Then, we analyze the obtained results and calculate the constants of motion of the disk. Moreover, the description of the motion of a charged particle on the halo is shown in Section 5. Finally, we complete the paper with a discussion of the results in Section 6.

\section{Exact Solutions for Relativistic Magnetized Haloes Surrounding Thin Disks}

In this section we consider the conventional treatment of rotating galaxies modelled as a stationary thin disk and, correspondingly, we associate the magnetized halo with the region surrounding the disk. This is motivated from the fact that the magnetic field is remarkably noticed on spiral galaxies and can play a fundamental role in formation of arms. To do so, we formulate the distributional EinsteinMaxwell field equations assuming axial symmetry [60]. We also suppose that the derivatives of the metric and electromagnetic potential across the disk space-like hypersurface are discontinuous. To formulate the corresponding distributional form of the Einstein-Maxwell field equations, we introduce the usual cylindrical coordinates $x^{\alpha}=(t, r, z, \varphi)$ and assume that there exists an infinitesimally thin disk located at the hypersurface $z=0$. Accordingly, we identify the halo surrounding the disk with the positive $(z \geq 0)$ and negative $(z \leq 0)$ regions around the equatorial plane $z=0$, denoted here by the superscripts " \pm ," so that the metric and the electromagnetic potential can be written, respectively, as $g_{\alpha \beta}=g_{\alpha \beta}^{+} \theta(z)+g_{\alpha \beta}^{-}\{1-\theta(z)\}$ and $A_{\alpha}=A_{\alpha}^{+} \theta(z)+A_{\alpha}^{-}\{1-$ $\theta(z)\}$. Here $\theta(z)$ denotes the Heaviside distribution. As a consequence, the Ricci tensor reads

$$
R_{\alpha \beta}=R_{\alpha \beta}^{+} \theta(z)+R_{\alpha \beta}^{-}\{1-\theta(z)\}+H_{\alpha \beta} \delta(z),
$$

where $\delta(z)$ is the Dirac distribution and

$$
H_{\alpha \beta}=\frac{1}{2}\left\{\gamma_{\alpha}^{z} \delta_{\beta}^{z}+\gamma_{\beta}^{z} \delta_{\alpha}^{z}-\gamma_{\mu}^{\mu} \delta_{\alpha}^{z} \delta_{\beta}^{z}-g^{z z} \gamma_{\alpha \beta}\right\}
$$

with $\gamma_{\alpha \beta}=2 g_{\alpha \beta, z}$ and all the quantities are evaluated at $z=$ $0^{+}$. In agreement with (1) the energy-momentum tensor and the electric current density acquire the form

$$
\begin{gathered}
T_{\alpha \beta}=T_{\alpha \beta}^{+} \theta(z)+T_{\alpha \beta}^{-}\{1-\theta(z)\}+Q_{\alpha \beta} \delta(z), \\
J_{\alpha}=J_{\alpha}^{+} \theta(z)+J_{\alpha}^{-}\{1-\theta(z)\}+\mathscr{I}_{\alpha} \delta(z),
\end{gathered}
$$


where $T_{\alpha \beta}^{ \pm}$and $J_{\alpha}^{ \pm}$are, respectively, the energy-momentum tensors and electric current density of halo. Moreover, $Q_{\alpha \beta}$ and $\mathscr{I}_{\alpha}$ represent the part of the energy-momentum tensor and the electric current density of disk. The energymomentum tensor $T_{\alpha \beta}^{ \pm}$in (3a) is taken to be the sum of two distributional components, the purely electromagnetic (trace-free) part and a "material" (trace) part:

$$
T_{\alpha \beta}^{ \pm}=E_{\alpha \beta}^{ \pm}+M_{\alpha \beta}^{ \pm},
$$

where $E_{\alpha \beta}^{ \pm}$is the electromagnetic energy-momentum tensor

$$
E_{\alpha \beta}=F_{\alpha \nu} F_{\beta}^{\nu}-\frac{1}{4} g_{\alpha \beta} F_{\mu \nu} F^{\mu \nu}
$$

with $F_{\alpha \beta}=A_{\beta, \alpha}-A_{\alpha, \beta}$ and $M_{\alpha \beta}^{ \pm}$is an unknown "material" energy-momentum tensor to be obtained. Accordingly, the Einstein-Maxwell equations, in geometrized units such that $c=8 \pi G=\mu_{0}=\epsilon_{0}=1$, are equivalent to the system of equations

$$
\begin{aligned}
G_{\alpha \beta}^{ \pm} & =R_{\alpha \beta}^{ \pm}-\frac{1}{2} g_{\alpha \beta} R^{ \pm}=E_{\alpha \beta}^{ \pm}+M_{\alpha \beta}^{ \pm}, \\
H_{\alpha \beta}-\frac{1}{2} g_{\alpha \beta} H & =Q_{\alpha \beta}, \\
F_{ \pm ; \beta}^{\alpha \beta} & =J_{ \pm}^{\alpha}, \\
\left.F^{\alpha \beta}\right] n_{\beta} & =\mathscr{I}^{\alpha},
\end{aligned}
$$

where $H \equiv g^{\alpha \beta} H_{\alpha \beta}$, and semicolon ";" in the index indicates the covariant derivative with respect to the metric. The square brackets in expressions such as $\left[F^{\alpha \beta}\right]$ denote the jump of $F^{\alpha \beta}$ across the surface $z=0$ and $n_{\beta}$ denotes a unitary vector in the direction normal to it. To obtain a solution of the Einstein-Maxwell equations describing a system composed of a magnetized halo surrounding a rotating thin disk we will restrict ourselves to the case where the electric potential $A_{t}=0$.

To solve the Einstein-Maxwell equations (6a), (6b), (6c), and (6d) we assume the conformastationary spacetime given by the line element

$$
d s^{2}=-e^{2 \phi}(d t+\omega d \varphi)^{2}+e^{-2 \beta \phi}\left(d r^{2}+d z^{2}+r^{2} d \varphi^{2}\right),
$$

where $\phi$ depends only on $r$ and $z$ and $\beta$ is an arbitrary real constant. We also assume that the magnetic potential $A_{\varphi}$ is time independent. Accordingly, by computing the Einstein tensor $G_{\alpha \beta}$ explicitly from line element (7) and electromagnetic energy-momentum tensor (5), we obtain for the nonzero components of the energy-momentum tensor of the halo (EMTH) $M_{\alpha \beta}^{ \pm}=E_{\alpha \beta}^{ \pm}-G_{\alpha \beta}^{ \pm}$:

$$
\begin{aligned}
M_{t t}^{ \pm} & =-e^{2(1+\beta) \phi}\left\{\beta^{2} \nabla \phi \cdot \nabla \phi-2 \beta \nabla^{2} \phi\right. \\
& \left.+\frac{1}{2} r^{-2} e^{2 \beta \phi} \nabla A_{\varphi} \cdot \nabla A_{\varphi}-\frac{3}{4} r^{-2} e^{2(1+\beta) \phi} \nabla \omega \cdot \nabla \omega\right\},
\end{aligned}
$$

$$
\begin{aligned}
M_{t \varphi}^{ \pm} & =e^{2(1+\beta) \phi}\left\{\frac{\beta}{2} \nabla \omega \cdot \nabla \phi+\frac{3}{4} r^{-2} e^{2(1+\beta) \phi} \omega \nabla \omega \cdot \nabla \omega\right. \\
& -\beta^{2} \omega \nabla \phi \cdot \nabla \phi+2 \beta \omega \nabla^{2} \phi+\frac{3}{2} \nabla \omega \cdot \nabla \phi \\
& \left.-\frac{1}{2} r^{-2} e^{2 \beta \phi} \omega \nabla A_{\varphi} \cdot \nabla A_{\varphi}+\frac{1}{2} \nabla^{2} \omega-r^{-1} \nabla \omega \cdot \nabla r\right\}, \\
M_{r r}^{ \pm} & =(1-\beta) \nabla^{2} \phi-(1-\beta) \phi_{, r r}+\left(\beta^{2}-2 \beta\right) \phi_{, r}^{2} \\
& +\phi_{, z}^{2}-\frac{1}{2} r^{-2} e^{2 \beta \phi}\left(A_{\varphi, r}^{2}-A_{\varphi, z}^{2}\right)+\frac{1}{4} \\
& \cdot r^{-2} e^{2(1+\beta) \phi}\left(\omega_{, r}^{2}-\omega_{, z}^{2}\right), \\
M_{r z}^{ \pm} & =\frac{1}{2} r^{-2} e^{2(1+\beta) \phi} \omega_{, r} \omega_{, z}-\left(1-\beta^{2}+2 \beta\right) \phi_{, r} \phi_{, z}-(1 \\
& -\beta) \phi_{, r z}-r^{-2} e^{2 \beta \phi} A_{\varphi, r} A_{\varphi, z}, \\
M_{z z}^{ \pm} & =-\frac{1}{4} r^{-2} e^{2(1+\beta) \phi}\left(\omega_{, r}^{2}-\omega_{, z}^{2}\right)+\phi_{, r}^{2}-(1-\beta) \phi_{, z z} \\
& +(1-\beta) \nabla^{2} \phi+\left(\beta^{2}-2 \beta\right) \phi_{, z}^{2}+\frac{1}{2} r^{-2} e^{2 \beta \phi}\left(A_{\varphi, r}^{2}\right. \\
& \left.-A_{\varphi, z}^{2}\right), \\
& \cdot \nabla r+(3+\beta) \omega \nabla \omega \cdot \nabla \phi-\frac{1}{2} r^{-2} e^{2 \beta \phi} \omega^{2} \nabla A_{\varphi} \\
M_{\varphi \varphi}^{ \pm} & =r^{2} \nabla \phi \cdot \nabla \phi+(1-\beta) r^{2} \nabla^{2} \phi-(1-\beta) r \nabla \phi \cdot \nabla r \\
& -\frac{1}{2} e^{2 \beta \phi} \nabla A_{\varphi} \cdot \nabla A_{\varphi} \\
& +e^{2(1+\beta) \phi}\left\{\frac{1}{4}\left(1+3 r^{-2} e^{2 \beta \phi} \omega^{2}\right) \nabla \omega \cdot \nabla \omega\right. \\
& \beta^{2} \nabla \phi+2 \beta \omega^{2} \nabla^{2} \phi+\omega \nabla^{2} \omega-2 r^{-1} \omega \nabla \omega \\
&
\end{aligned}
$$

Moreover, from (6c) the nonzero components of the electric current density on the halo have the form

$$
\begin{aligned}
& J_{ \pm}^{t}=e^{-(1-3 \beta) \phi} \nabla \cdot\left\{\omega r^{-2} e^{(1+\beta) \phi} \nabla A_{\varphi}\right\}, \\
& J_{ \pm}^{\varphi}=e^{-(1-3 \beta) \phi} \nabla \cdot\left\{r^{-2} e^{(1+\beta) \phi} \nabla A_{\varphi}\right\},
\end{aligned}
$$

where all the quantities depend on $r$ and $z$.

The discontinuity in the $z$-direction of $Q_{\alpha \beta}$ and $\mathcal{J}^{\alpha}$ defines, respectively, the surface energy-momentum tensor (SEMT) $S_{\alpha \beta}$ and the surface electric current density (SECD) $\mathcal{F}^{\alpha}$ of the disk, more precisely

$$
\begin{aligned}
& S_{\alpha \beta} \equiv \int Q_{\alpha \beta} \delta(z) d s_{n}=\sqrt{g_{z z}} Q_{\alpha \beta}, \\
& \mathscr{J}^{\alpha} \equiv \int \mathscr{I}^{\alpha} \delta(z) d s_{n}=\sqrt{g_{z z}} \mathscr{I}^{\alpha},
\end{aligned}
$$

where $d s_{n}=\sqrt{g_{z z}} d z$ is the "physical measure" of length in the direction normal to the $z=0$ surface. Accordingly, for 
metric (7), the nonzero components of $S_{\alpha \beta}$ and $\mathscr{J}^{\alpha}$ are given by

$$
\begin{aligned}
S_{t t} & =4 \beta e^{(2+\beta) \phi} \phi_{, z}, \\
S_{t \varphi} & =e^{(2+\beta) \phi}\left(4 \beta \omega \phi_{, z}+\omega_{, z}\right), \\
S_{r r} & =2(1-\beta) e^{-\beta \phi} \phi_{, z}, \\
S_{\varphi \varphi} & =e^{(2+\beta) \phi}\left\{\left(4 \beta \omega^{2}+2(1-\beta) r^{2} e^{-2(1+\beta) \phi}\right) \phi_{, z}\right. \\
& \left.+2 \omega \omega_{, z}\right\}, \\
\mathscr{J}^{t} & =r^{-2} e^{3 \beta \phi} \omega\left[A_{\varphi, z}\right], \\
\mathscr{g}^{\varphi} & =-r^{-2} e^{3 \beta \phi}\left[A_{\varphi, z}\right],
\end{aligned}
$$

respectively. Note that in (11a), (11b), (11c), and (11d) and (12a) and $(12 \mathrm{~b})$ all the quantities are evaluated on the surface of the $\operatorname{disk}(z=0)$.

In order to reduce the complexity of the last field equation systems we assume that the halo's electric current density vanishes (i.e., $J_{ \pm}^{t}=J_{ \pm}^{\varphi}=0$ in (9a) and (9b)), and it turns out that the magnetic potential and the metric functions $\phi$ and $\omega$ become completely determined in terms of an arbitrary harmonic function $U(r, z)$ as follows (see $[54,56]$ for more details):

$$
\begin{aligned}
A_{\varphi, r} & =-\frac{1}{k} r U_{, z}, \\
A_{\varphi, z} & =\frac{1}{k} r U_{, r}, \\
(\beta+1) \phi & =-\ln (1-U), \\
\omega & =k_{\omega} U,
\end{aligned}
$$

with $k$ and $k_{\omega}$ arbitrary constants. Since the nonzero components of the EMTD and EMTH and the electric current density directly depend on the metric functions and magnetic potential, we observe that the entire solution is determined by a single harmonic function.

\section{Exact Relativistic Model for Magnetized Disk-Haloes}

So far, by using the inverse method and the distributional formulation of the Einstein-Maxwell equations, we have obtained the separate energy-momentum tensor of the disk and halo. In addition, we have discussed a method to determine its components in terms of an arbitrary harmonic function. Now, the behavior of the energy-momentum tensors obtained must be investigated to find what conditions must be imposed on the solutions and the parameters that appear in the disk-haloes models in such a way that it can describe reasonably physical sources. We will now study the possible features of the disk by assuming that it is possible to express its energy-momentum tensor in the canonical form:

$$
S_{\alpha \beta}=(\mu+P) V_{\alpha} V_{\beta}+P g_{\alpha \beta}+\mathbb{Q}_{\alpha} V_{\beta}+\mathbb{Q}_{\beta} V_{\alpha}+\Pi_{\alpha \beta},
$$

where $\mathbb{Q}_{\alpha} V^{\alpha}=\mathscr{Q}^{\alpha} V_{\alpha}=0, \alpha=(t, r, \varphi)$, and all the quantities are evaluated in $z=0^{+}$. Similarly, we assume that it is possible to express the energy-momentum tensor of the halo in the canonical form:

$$
\begin{aligned}
M_{\alpha \beta}^{ \pm}= & \left(\mu^{ \pm}+P^{ \pm}\right) V_{\alpha} V_{\beta}+P^{ \pm} g_{\alpha \beta}+\mathcal{Q}_{\alpha}^{ \pm} V_{\beta}+\mathcal{Q}_{\beta}^{ \pm} V_{\alpha} \\
& +\Pi_{\alpha \beta}^{ \pm},
\end{aligned}
$$

where $\mathbb{Q}_{\alpha}^{ \pm} V^{\alpha}=Q^{ \pm \alpha} V_{\alpha}=0, \alpha=(t, r, z, \varphi)$, and all the quantities depend on $r$ and $z$. Consequently, we can say that the disk and halo are constituted by some mass-energy distributions described by the energy-momentum tensors (14) and (15), respectively. $V^{\alpha}$ is the four-velocity of a certain observer. Correspondingly, $\mu, P, Q_{\alpha}$, and $\Pi_{\alpha \beta}$ are then the energy density, the isotropic pressure, the heat flux, and the anisotropic tensor on the surface of the disk. Analogously, $\mu^{ \pm}$, $P^{ \pm}, Q_{\alpha}^{ \pm}$, and $\Pi_{\alpha \beta}^{ \pm}$are then the energy density, the isotropic pressure, the heat flux, and the anisotropic tensor on the halo, respectively. Thus, it is straightforward to see that for the halo we have

$$
\begin{aligned}
\mu^{ \pm} & =M_{\alpha \beta}^{ \pm} V^{\alpha} V^{\beta}, \\
P^{ \pm} & =\frac{1}{3} \mathscr{H}^{\alpha \beta} M_{\alpha \beta}^{ \pm}, \\
Q_{\alpha}^{ \pm} & =-\mu^{ \pm} V_{\alpha}-M_{\alpha \beta}^{ \pm} V^{\beta}, \\
\Pi_{\alpha \beta}^{ \pm} & =\mathscr{H}_{\alpha}{ }^{\mu} \mathscr{H}_{\beta}{ }^{\nu}\left(M_{\mu \nu}^{ \pm}-P^{ \pm} \mathscr{H}_{\mu \nu}\right),
\end{aligned}
$$

where the projection tensor is defined by $\mathscr{H}_{\mu \nu} \equiv g_{\mu \nu}+V_{\mu} V_{\nu}$ and all the quantities depend on $r$ and $z$, whereas for the disk we have

$$
\begin{aligned}
\mu & =S_{\alpha \beta} V^{\alpha} V^{\beta}, \\
P & =\frac{1}{3} \mathscr{H}^{\alpha \beta} S_{\alpha \beta}, \\
Q_{\alpha} & =-\mu V_{\alpha}-S_{\alpha \beta} V^{\beta}, \\
\Pi_{\alpha \beta} & =\mathscr{H}_{\alpha}{ }^{\mu} \mathscr{H}_{\beta}{ }^{\nu}\left(S_{\mu \nu}-P \mathscr{H}_{\mu \nu}\right),
\end{aligned}
$$

where all the quantities are evaluated in $z=0^{+}$. It is easy to note that by choosing the angular velocity to be zero in (A.7) we have then a fluid comoving in our coordinates system. Hence, we may introduce a suitable reference frame in terms of the local observers tetrads (A.3a), (A.3b), (A.3c), and (A.3d) and (A.4a), (A.4b), (A.4c), and (A.4d) in the form

$$
\left\{V^{\alpha}, I^{\alpha}, K^{\alpha}, Y^{\alpha}\right\} \equiv\left\{h_{(t)}{ }^{\alpha}, h_{(r)}{ }^{\alpha}, h_{(z)}{ }^{\alpha}, h_{(\varphi)}{ }^{\alpha}\right\},
$$

with the corresponding dual tetrad

$$
\left\{V_{\alpha}, I_{\alpha}, K_{\alpha}, Y_{\alpha}\right\} \equiv\left\{-h^{(t)}, h_{\alpha}^{(r)}, h_{\alpha}^{(z)}, h_{\alpha}^{(\varphi)}\right\} .
$$

Since the SECD of the disk $\mathcal{F}^{\alpha}$ can be also written in the canonical form $\mathcal{g}^{\alpha}=\sigma V^{\alpha}+j Y^{\alpha}, \sigma$ can be interpreted as the surface electric charge density and $j$ as the "current of 
magnetization" of the disk. A direct calculation shows that the surface electric charge density $\sigma=0$, whereas the "current of magnetization" of the disk is given by $j=-r^{-1} e^{2 \beta \phi}\left[A_{\varphi, z}\right]$, where, as above, $\left[A_{\varphi, z}\right]$ denotes the jump of the $z$-derivative of the magnetic potential across the disk and, all quantities are evaluated on the disk.

By using the results obtained in the precedent section, we can write the surface energy density of the disk and the energy density of the halo can written as

$$
\mu(r)=\frac{4 \beta U_{, z}}{(\beta+1)(1-U)^{(2 \beta+1) /(\beta+1)}},
$$

$$
\begin{gathered}
\mu^{ \pm}(r, z)=\frac{\left(U_{, r}^{2}+U_{, z}^{2}\right) e^{2(1+2 \beta) \phi}}{(1+\beta)^{2} r^{2}}\left\{\left(2 \beta+\beta^{2}\right) r^{2}\right. \\
\left.-\frac{(1+\beta)^{2}}{2 k^{2}} r^{2} e^{-2 \phi}+\frac{3 k_{\omega}^{2}(1+\beta)^{2}}{4}\right\},
\end{gathered}
$$

respectively. Moreover, we have a barotropic equation of state on the surface of the disk, which can be given by $P(r)=\eta \mu$, with $\eta=(1-\beta) / 3 \beta$, in such a way that we can use the energy conditions and the causality requirement for the speed of sound on the disk to obtain the physical range of possible values of the parameter $\beta$. Analogously, the pressure of the halo is as follow: $P^{ \pm}(r, z)=\Theta \mu^{ \pm}(r, z)$, where

$$
\Theta:=\frac{\left(4-2 \beta-\beta^{2}\right) r^{2}-\left((1+\beta)^{2} / 2 k^{2}\right) r^{2} e^{-2 \phi}+\left(k_{\omega}^{2}(1+\beta)^{2} / 4\right)\left(1+3 k_{\omega}^{2} r^{-2} U^{2} e^{2 \beta \phi}\left(1-e^{2 \phi}\right)\right)}{3\left(\left(2 \beta+\beta^{2}\right) r^{2}-\left((1+\beta)^{2} / 2 k^{2}\right) r^{2} e^{-2 \phi}+3 k_{\omega}^{2}(1+\beta)^{2} / 4\right)},
$$

in such a way that the pressure of the halo depends not only on the energy density but also on the gravitational and magnetic fields through the function $\Theta$. The heat function of the disk is given by

$$
Q_{\alpha}(r)=-\frac{k_{\omega} U_{, z}}{1-U} \delta_{\alpha}^{\varphi}
$$

Similarly, by inserting (15) into (16a), (16b), (16c), and (16d) we obtain for the heat flux of the halo

$$
\begin{aligned}
\mathcal{Q}_{\alpha}^{ \pm} & =\frac{k_{\omega} e^{(1+2 \beta) \phi}}{2(1+\beta) r}\left\{2(1+\beta) U_{, r}\right. \\
& \left.-(3+\beta) r\left(U_{, r}^{2}+U_{, z}^{2}\right) e^{(1+\beta) \phi}\right\} \delta_{\alpha}^{\varphi} .
\end{aligned}
$$

The nonzero components of the anisotropic tensor of the disk read $\Pi_{\varphi \varphi}(r)=r^{2} \Pi_{r r}(r)$, where

$$
\Pi_{r r}(r)=\frac{2(1-\beta) U_{, z}}{3(1+\beta)(1-U)^{1 /(1+\beta)}} .
$$

Moreover, it is easy to see that the anisotropic tensor of the halo reads

$$
\Pi_{\alpha \beta}^{ \pm}=P_{r}^{ \pm} I_{\alpha} I_{\beta}+P_{z}^{ \pm} K_{\alpha} K_{\beta}+P_{\varphi}^{ \pm} Y_{\alpha} Y_{\beta}+2 P_{T}^{ \pm} I_{(\alpha} K_{\beta)},
$$

where, $2 I_{(\alpha} K_{\beta)}=I_{\alpha} K_{\beta}+I_{\beta} K_{\alpha}$, as in the usual manner and

$$
\begin{aligned}
& P_{r}^{ \pm}=e^{2 \beta \phi} \Pi_{r r}^{ \pm}, \\
& P_{z}^{ \pm}=e^{2 \beta \phi} \Pi_{z z}^{ \pm}, \\
& P_{\varphi}^{ \pm}=\frac{e^{2 \beta \phi}}{r^{2}} \Pi_{\varphi \varphi}^{ \pm}, \\
& P_{T}^{ \pm}=e^{2 \beta \phi} \Pi_{r z}^{ \pm},
\end{aligned}
$$

$$
\begin{aligned}
& \Pi_{r r}^{ \pm}=\frac{e^{2(1+\beta) \phi}}{3(1+\beta)^{2} r^{2}}\left\{\left(\frac{k_{\omega}^{2}(1+\beta)^{2}}{2}\right.\right. \\
& +\frac{2(1+\beta)^{2}}{k^{2}} r^{2} e^{-2 \phi}-4\left(1+\beta-\beta^{2}\right) r^{2} \\
& \left.-\frac{3 k_{\omega}^{4}(1+\beta)^{2}}{4} r^{-2} U^{2} e^{2 \beta \phi}\left(1-e^{2 \phi}\right)\right) U_{r}^{2} \\
& +\left(-k_{\omega}^{2}(1+\beta)^{2}-\frac{(1+\beta)^{2}}{k^{2}} r^{2} e^{-2 \phi}\right. \\
& +2\left(1+\beta-\beta^{2}\right) r^{2} \\
& \left.-\frac{3 k_{\omega}^{4}(1+\beta)^{2}}{4} r^{-2} U^{2} e^{2 \beta \phi}\left(1-e^{2 \phi}\right)\right) U_{z}^{2}-3(1 \\
& \left.\left.-\beta^{2}\right) r^{2} e^{-(1+\beta) \phi} U_{, r r}\right\} \\
& \Pi_{z z}^{ \pm}=\frac{e^{2(1+\beta) \phi}}{3(1+\beta)^{2} r^{2}}\left\{\left(-k_{\omega}^{2}(1+\beta)^{2}\right.\right. \\
& -\frac{(1+\beta)^{2}}{k^{2}} r^{2} e^{-2 \phi}+2\left(1+\beta-\beta^{2}\right) r^{2} \\
& \left.-\frac{3 k_{\omega}^{4}(1+\beta)^{2}}{4} r^{-2} U^{2} e^{2 \beta \phi}\left(1-e^{2 \phi}\right)\right) U_{r}^{2} \\
& +\left(\frac{k_{\omega}^{2}(1+\beta)^{2}}{2}+\frac{2(1+\beta)^{2}}{k^{2}} r^{2} e^{-2 \phi}\right. \\
& -4\left(1+\beta-\beta^{2}\right) r^{2}
\end{aligned}
$$




$$
\begin{aligned}
& \left.-\frac{3 k_{\omega}^{4}(1+\beta)^{2}}{4} r^{-2} U^{2} e^{2 \beta \phi}\left(1-e^{2 \phi}\right)\right) U_{z}^{2}-3(1 \\
& \left.\left.-\beta^{2}\right) r^{2} e^{-(1+\beta) \phi} U_{, z z}\right\}, \\
\Pi_{\varphi \varphi}^{ \pm} & =\frac{\left(U_{, r}^{2}+U_{, z}^{2}\right) e^{2(1+\beta) \phi}}{3(1+\beta)^{2}}\left\{2\left(1+\beta-\beta^{2}\right) r^{2}\right. \\
& -\frac{(1+\beta)^{2}}{k^{2}} r^{2} e^{-2 \phi}+\frac{k_{\omega}^{2}(1+\beta)^{2}}{2}(1 \\
& \left.\left.+3 k_{\omega}^{2} r^{-2} U^{2} e^{2 \beta \phi}\left(1-e^{2 \phi}\right)\right)\right\}-\frac{(1-\beta)}{1+\beta} \\
& \cdot r e^{(1+\beta) \phi} U_{, r}, \\
+ & \left.\left.\frac{k_{\omega}^{2}(1+\beta)^{2}}{2 r^{2}}\right) U_{, r} U_{, z}-\left(1-\beta^{2}\right) e^{-(1+\beta) \phi} U_{, r z}\right\} \\
\Pi_{r z}^{2(1+\beta \phi)} & =\frac{(1+\beta)^{2}}{(1+\beta)^{2}} e^{-2 \phi}
\end{aligned}
$$

Notice that $\mathscr{P}^{ \pm} \equiv P_{r}^{ \pm}+P_{z}^{ \pm}+P_{\varphi}^{ \pm}=0$ and, consequently, the trace $\Pi_{\alpha}^{ \pm \alpha}=0$. We have obtained expressions for the energy, pressure, and the other quantities characterizing the dynamic of the halo. All the dynamic quantities have been expressed in terms of an arbitrary $U(r, z)$ harmonic function. Finally, as we know, the electric current density of the halo is zero whereas it is easy to note that the magnetization current density on surface of the disk is

$$
j(r)=-\frac{\left[U_{, r}\right]}{k(1-U)^{3 \beta /(1+\beta)}} .
$$

It is important to remark that $k_{\omega}$ is a defining constant in (23) and (24). Indeed, when $k_{\omega}=0$ the heat flux functions $Q_{\alpha}$ and $Q_{\alpha}^{ \pm}$vanish, a feature of the static systems. Because we used the inverse method, no "a priori" restriction is imposed on the physical properties of the material constituting the disk and halo. The nonzero components of the energy-momentum tensors of the disk and halo result from "the nature" of the chosen metric and the corresponding solutions. So, in our case, the nonzero components $S_{r r}$ and $S_{t \varphi}$ are conditioned by the parameter $\beta$ and the metric function $\omega$ in such a way that when $\beta=1$ the component $S_{r r}$ vanishes, whereas $S_{t \varphi}=0$ when $\omega$ vanishes. The decomposition of the energymomentum tensor of the disk-halo system into (14) and (15) was chosen with the aim of describing the SEMT and EMTH by the more general fluid model. Hence, the heat flux appears here in a "natural" way as a function determined by the metric function $\omega$ and, consequently, by the "rotation." Unfortunately, as we can see from (17c) and (16c), this function is oriented along the closed circular orbits and thus its physical interpretation is unclear. It is an issue that remains unanswered in this paper but should be addressed in the future.

\section{Rotating Kuzmin-Like Disk with Magnetized Haloes}

As an example of application of the formalism described in the precedent sections, we now consider the magnetized haloes surrounding the rotating disks generated by a generalization of the Kuzmin-disk potential in the form [57, 61]

$$
\begin{aligned}
U=-\sum_{n=0}^{N} \frac{b_{n} P_{n}(z / R)}{R^{n+1}}, & \\
& P_{n}\left(\frac{z}{R}\right)=(-1)^{n} \frac{R^{n+1}}{n !} \frac{\partial^{n}}{\partial z^{n}}\left(\frac{1}{R}\right),
\end{aligned}
$$

where $P_{n}=P_{n}(z / R)$ is the Legendre polynomial in cylindrical coordinates that was derived in the present form by a direct comparison of the Legendre polynomial expansion of the generating function with a Taylor series expansion of $1 / R$ [62], being $R^{2} \equiv r^{2}+z^{2}$ and $b_{n}$ arbitrary constant coefficients. The corresponding magnetic potential, obtained from (13a) and $(13 b)$, is

$$
A_{\varphi}=-\frac{1}{k} \sum_{n=0}^{N} b_{n} \frac{(-1)^{n}}{n !} \frac{\partial^{n}}{\partial z^{n}}\left(\frac{z}{R}\right)
$$

where we have imposed $A_{\varphi}(0, z)=0$ in order to preserve the regularity of the axis of symmetry. Next, to introduce the corresponding discontinuity in the first-order derivatives of the metric potential and the magnetic potential required to define the disk we perform the transformation $z \rightarrow|z|+a$. It is worth noting that, for the two first members of the family of solutions ( $N=0$ and $N=1)$ we have

$$
\begin{aligned}
& U_{0}=-\frac{\widetilde{b}_{0}}{\sqrt{\widetilde{r}^{2}+(|\widetilde{z}|+1)^{2}}}, \\
& \widetilde{A}_{\varphi 0}=-\frac{\tilde{b}_{0}(|\tilde{z}|+1)}{k \sqrt{\widetilde{r}^{2}+(|\widetilde{z}|+1)^{2}}} \\
& \widetilde{A}_{\varphi 1}=-\frac{\widetilde{b}_{0}(|\tilde{z}|+1)}{k \sqrt{\widetilde{r}^{2}+(|\tilde{z}|+1)^{2}}}\{1 \\
& \left.-\frac{\tilde{b}_{1} \widetilde{r}^{2}}{\widetilde{b}_{0}(|\tilde{z}|+1)\left((|\widetilde{z}|+1)^{2}+\widetilde{r}^{2}\right)}\right\}, \\
& U_{1}=-\frac{\tilde{b}_{0}}{\sqrt{\widetilde{r}^{2}+(|\tilde{z}|+1)^{2}}}\left\{1+\frac{\tilde{b}_{1}(|\tilde{z}|+1)}{\widetilde{b}_{0}\left((|\tilde{z}|+1)^{2}+\widetilde{r}^{2}\right)}\right\},
\end{aligned}
$$


respectively, where $\tilde{b}_{0}=b_{0} / a$ and $\tilde{b}_{1}=b_{1} / a^{2}$ whereas $\tilde{r}=r / a$ and $\widetilde{z}=z / a$; moreover $\widetilde{A}_{\varphi}=A_{\varphi} / a$. For the two first members of the family of solutions the surface energy density of the disks can be written as

$$
\begin{aligned}
& \tilde{\mu}_{0}=\frac{4 \beta \tilde{b}_{0}}{(1+\beta)\left(\widetilde{r}^{2}+1\right)^{(\beta+2) /(2 \beta+2)}\left(\widetilde{b}_{0}+\sqrt{\widetilde{r}^{2}+1}\right)^{(2 \beta+1) /(\beta+1)}}, \\
& \tilde{\mu}_{1}=\frac{4 \beta\left\{\left(\widetilde{b}_{0}-\widetilde{b}_{1}\right) \tilde{r}^{2}+\widetilde{b}_{0}+2 \widetilde{b}_{1}\right\}}{(1+\beta)\left(\widetilde{r}^{2}+1\right)^{(2-\beta) /(2 \beta+2)}\left\{\left(\widetilde{r}^{2}+1\right)^{3 / 2}+\tilde{b}_{0}\left(\widetilde{r}^{2}+1\right)+\tilde{b}_{1}\right\}^{(2 \beta+1) /(\beta+1)}},
\end{aligned}
$$

respectively. Similarly, for the two first members of the family we have the heat flux of the disks

$$
Q_{\alpha 0}=\frac{\widetilde{b}_{0} k_{\omega} \delta_{\alpha}^{\varphi}}{\sqrt{\widetilde{r}^{2}+1}+\widetilde{b}_{0}},
$$

$$
Q_{\alpha 1}=\frac{k_{\omega} \delta_{\alpha}^{\varphi}\left(\widetilde{b}_{0}\left(\widetilde{r}^{2}+1\right)+\widetilde{b}_{1}\right)}{\left(\widetilde{r}^{2}+1\right)^{3 / 2}+\widetilde{b}_{0}\left(\widetilde{r}^{2}+1\right)+\widetilde{b}_{1}}
$$

and the corresponding anisotropic tensor

$$
\begin{aligned}
& \widetilde{\Pi}_{r r 0}=\frac{2(1-\beta) \widetilde{b}_{0}}{3(1+\beta)\left(\sqrt{\widetilde{r}^{2}+1}+\widetilde{b}_{0}\right)^{1 /(1+\beta)}\left(\widetilde{r}^{2}+1\right)^{(3 \beta+2) /(2+2 \beta)}}, \\
& \widetilde{\Pi}_{r r 1}=\frac{2(1-\beta)\left(\left(\widetilde{b}_{0}-\widetilde{b}_{1}\right) \widetilde{r}^{2}+\widetilde{b}_{0}+2 \widetilde{b}_{1}\right)}{3(1+\beta)\left\{\left(\widetilde{r}^{2}+1\right)^{3 / 2}+\widetilde{b}_{0}\left(\widetilde{r}^{2}+1\right)+\widetilde{b}_{1}\right\}^{1 /(1+\beta)}\left(\widetilde{r}^{2}+1\right)^{(5 \beta+2) /(2+2 \beta)}} .
\end{aligned}
$$

As we know, other quantities are $P=(1-\beta) \mu /(3 \beta)$ and $\Pi_{\varphi \varphi}=$ $r^{2} \Pi_{r r}$. In the last expressions we have used the dimensionless expressions $\widetilde{\mu}=a \mu, \widetilde{\Pi}_{\varphi \varphi}=a \Pi_{r r}$. Finally, for the two first members of the family we have the current of magnetization as

$$
\begin{aligned}
& \widetilde{j_{0}}=-\frac{2 \widetilde{b}_{0} \widetilde{r}}{k\left(\widetilde{r}^{2}+1\right)^{(3+\beta) /(2+2 \beta)}\left(\widetilde{b}_{0}+\sqrt{\widetilde{r}^{2}+1}\right)^{2 \beta /(\beta+1)}}, \\
& \widetilde{j}_{1} \\
& =-\frac{2 \widetilde{r}\left(\widetilde{b}_{0}\left(\widetilde{r}^{2}+1\right)+3 \widetilde{b}_{1}\right)}{k\left(\widetilde{r}^{2}+1\right)^{(5-\beta) /(2+2 \beta)}\left\{\left(\widetilde{r}^{2}+1\right)^{3 / 2}+\widetilde{b}_{0}\left(\widetilde{r}^{2}+1\right)+\widetilde{b}_{1}\right\}^{2 \beta /(1+\beta)}},
\end{aligned}
$$

where $\tilde{j}=a j$ and we first have assumed that the $z$-derivative of the magnetic potential presents a finite discontinuity through the disk. In fact, as we have said above, the derivatives of $U$ and $A_{\varphi}$ are continuous functions across the surface of the disk. We artificially introduce the discontinuity through the transformation $z \rightarrow|z|+a$.

It is worth noticing that the mass surface density as well as the isotropic pressure of the disk decays very rapidly (as $1 / r^{3}$ and $1 / r^{5}$ for $N=0$ and $N=1$, resp.) indicating that the above solution can be associated with a disk with a finite energymomentum distribution. In every case, the characteristic size can be adjusted through the parameters $b_{0}$ and $b_{1}$ of the solutions. Moreover, a simple calculation of the curvature invariants reveals that the solution is asymptotically flat and singularity-free.

To illustrate the results corresponding to the principal quantities describing the halo in Figure 1, we show the behavior of energy densities $\mu^{ \pm}$on the halo as a function of $r$ and $z$. In each case, we plot $\mu_{0}^{ \pm}(r, z)$ (Figure $1(\mathrm{a})$ ) and $\mu_{1}^{ \pm}(r, z)$ (Figure 1(b)) for the indicated values of the parameters. It can be seen that the energy density is everywhere positive and vanishes sufficiently fast as $r$ increases.

In Figure 2, we show the behavior of pressure $P^{ \pm}$on the halo as a function of $r$ and $z$. In each case, we plot $P_{0}^{ \pm}(r, z)$ (Figure $2(\mathrm{a})$ ) and $P_{1}^{ \pm}(r, z)$ (Figure 2(b)) for the indicated values of the parameters. We can see that pressure is always positive and behaves as the energy density of the halo. Thus, we can see that the behavior of these quantities is in agreement with the results published in [53]. Moreover, we also computed these functions for other values of the parameters within the allowed range and in all cases we have found a similar behavior.

4.1. The Constants of Motion. To proceed further, we evaluate the constants of motion. Therefore, from (13c) we have

$$
\phi=\frac{1}{1+\beta} \ln \left(\frac{1}{1-U}\right) .
$$




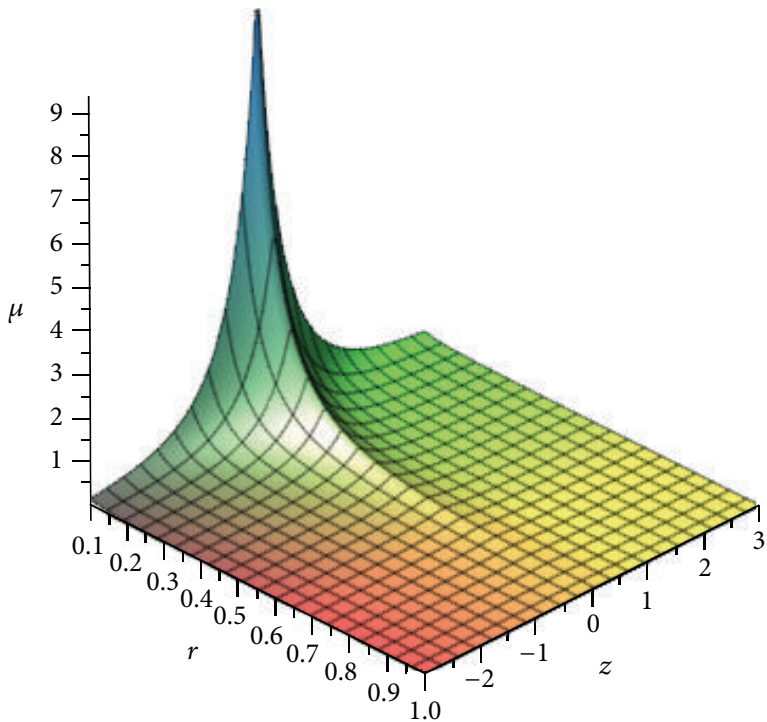

(a)

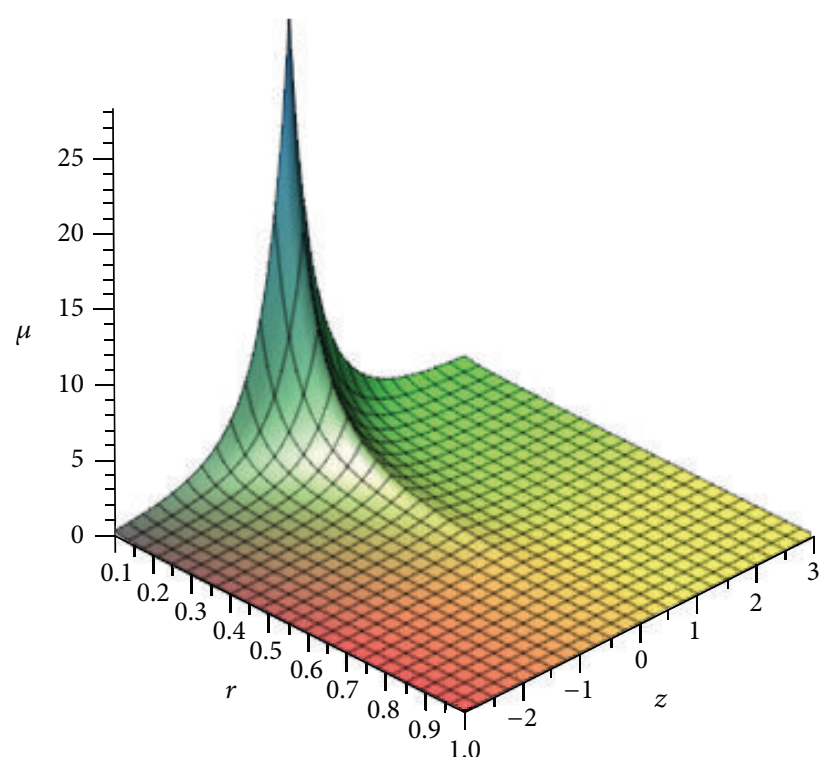

(b)

FIGURE 1: Surface plots of the energy densities (a) $\mu_{0}^{ \pm}$and (b) $\mu_{1}^{ \pm}$on the halo as functions depending on $r$ and $z$ with parameters $a=b_{0}=b_{1}=$ $k=k_{\omega}=1$ and $\beta=0.75$.

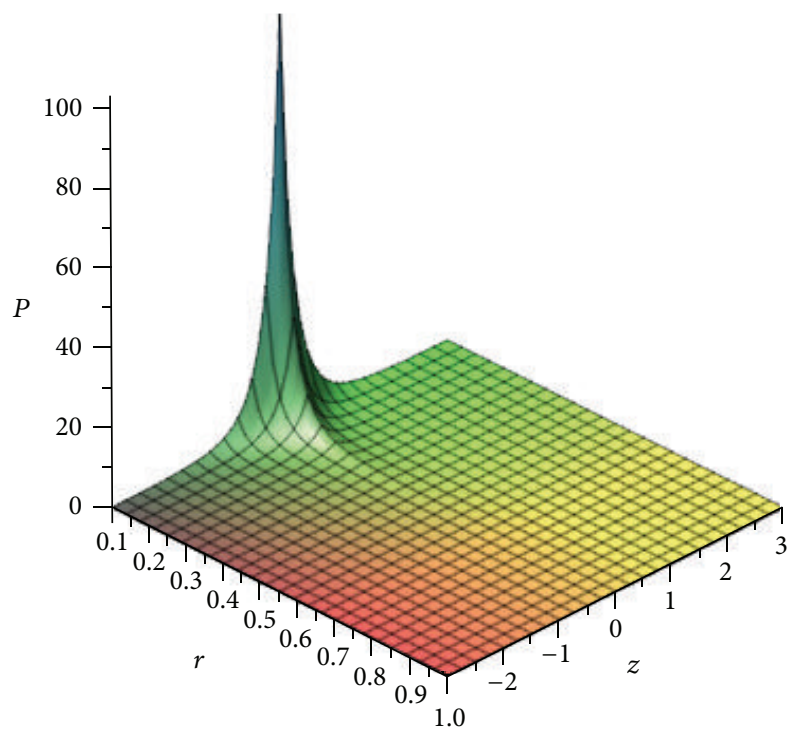

(a)

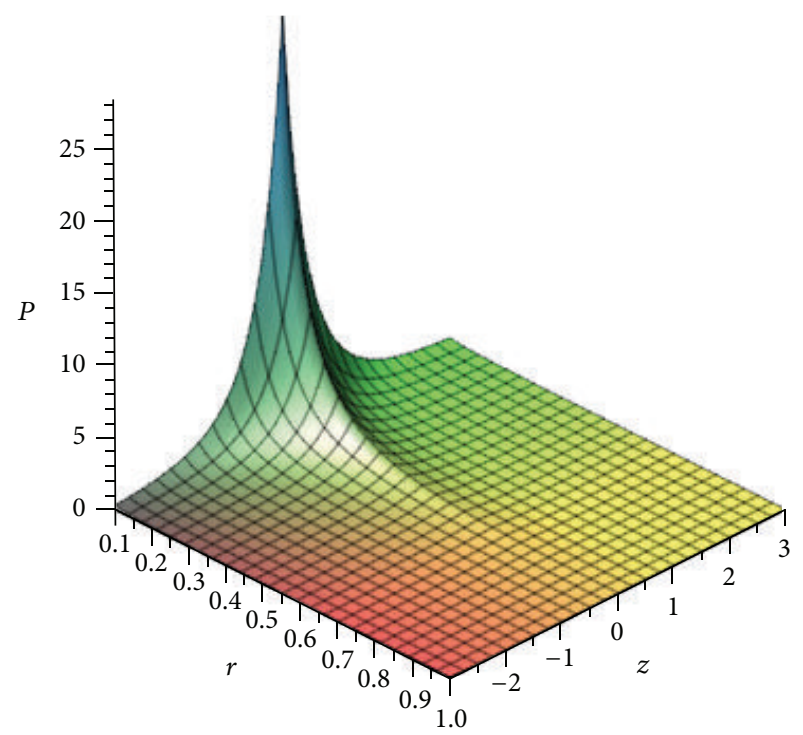

(b)

Figure 2: Surface plots of the pressures (a) $P_{0}^{ \pm}$and (b) $P_{1}^{ \pm}$on the halo as functions depending on $r$ and $z$ with parameters $a=b_{0}=b_{1}=k=$ $k_{\omega}=1$ and $\beta=0.75$.

Then, for solution (32a) we may write

$$
\phi_{0}=\frac{1}{1+\beta} \ln \left(\frac{\sqrt{\widetilde{R}^{2}+2|\tilde{z}|+1}}{\sqrt{\widetilde{R}^{2}+2|\tilde{z}|+1}+\widetilde{b}_{0}}\right) \text {, }
$$

where $\widetilde{R}^{2} \equiv \widetilde{r}^{2}+\widetilde{z}^{2}$. This follows that the metric potentials $g_{t t}$ and $g_{t \varphi}$ for $R \rightarrow \infty$ in the disk $(z=0)$ become

$$
g_{t t} \simeq-1+\frac{2 \tilde{b}_{0}}{(1+\beta) \widetilde{R}}-\frac{\widetilde{b}_{0}^{2}(3+\beta)}{(1+\beta)^{2} \widetilde{R}^{2}}+O\left(\frac{1}{\widetilde{R}^{3}}\right),
$$

$$
g_{t \varphi} \simeq \frac{k_{\omega} \widetilde{b}_{0}}{\widetilde{R}}-\frac{2 k_{\omega} \widetilde{b}_{0}^{2}}{(1+\beta) \widetilde{R}^{2}}+O\left(\frac{1}{\widetilde{R}^{3}}\right) .
$$

This implies, as is well known (see [41]), that the total massenergy of spacetime associated with the disk is

$$
M_{0}=\frac{b_{0}}{(1+\beta)} \text {. }
$$


On the other hand, in $(x, y, z)$ coordinates we find that

$$
\begin{aligned}
& g_{01} \simeq-\frac{k_{\omega} \widetilde{b}_{0} y}{\widetilde{R}^{3}}+O\left(\frac{1}{\widetilde{R}^{4}}\right), \\
& g_{02} \simeq \frac{k_{\omega} \widetilde{b}_{0} x}{\widetilde{R}^{3}}+O\left(\frac{1}{\widetilde{R}^{4}}\right), \\
& g_{03} \simeq O\left(\frac{1}{\widetilde{R}^{4}}\right) .
\end{aligned}
$$

As an application, we use the same procedure as in [63] and see that the angular momentum $L_{M 0}$ is in the $z$-direction and is given by

$$
L_{M 0}=\frac{1}{2} k_{\omega} b_{0} .
$$

According to (32b) the magnetic field is

$$
\mathbf{B}_{0}=-\frac{\tilde{b}_{0} \tilde{r}}{k\left(\widetilde{R}^{2}+2|\widetilde{z}|+1\right)^{3 / 2}}\left(\tilde{r} \mathbf{e}_{r}+\left(\tilde{z}+\frac{|\widetilde{z}|}{\widetilde{z}}\right) \mathbf{e}_{z}\right),
$$

where $\mathbf{e}_{\alpha}$ are unit basis vectors in cylindrical coordinates. Accordingly, by expressing the components of the magnetic field in Cartesian coordinates and taking the limit as $R \rightarrow \infty$ of $\mathbf{B}_{0}(x, y, z)$ and by using formula $(44.4)$ of Landau and Lifshits [64] we may conclude that the magnetic momentum may be written as

$$
L_{B 0}=\frac{b_{0}}{k}
$$

We thus see that constants $k$ and $k_{\omega}$ define the gyromagnetic ratio $L_{M 0} / L_{B 0}=\left(k k_{\omega}\right) / 2$.

\section{Motion of a Charged Test Particle in the Halo}

The motion of a test particle of charge $e$ and mass $m$ moving in the halo is described by the following Lagrangian density:

$$
\mathscr{L}=\frac{1}{2} g_{\alpha \beta} v^{\alpha} v^{\beta}+\frac{e}{m} A_{\alpha} x^{\alpha},
$$

where $g_{\alpha \beta}$ and $A_{\alpha}$ are, respectively, the components of the metric and electromagnetic potential, given here by (13a), (13b), (13c), and (13d).

The equations of motion of the test particle can be derived from (47) by using the Euler-Lagrange equation. Then,

$$
\frac{d v^{\alpha}}{d s}+\Gamma_{\beta \gamma}^{\alpha} v^{\beta} v^{\gamma}=\frac{e}{m} g^{\alpha \mu} F_{\mu \lambda} v^{\lambda} .
$$

The velocity of the particle as measured by the local observers is given by $v^{\alpha}=v^{t}\left(t^{\alpha}+\Omega \varphi^{\alpha}\right)$, where

$$
v^{t}=\frac{(1-U)^{1 /(1+\beta)}}{\left(1+k_{\omega} U \Omega\right) \sqrt{1-v^{2}}} .
$$

Here, the 3-velocity $v$ and the angular velocity $\Omega$ of the particle as measured by the local observers are given by

$$
\begin{array}{rl}
v= & \frac{r \Omega(1-U)}{1+k_{\omega} U \Omega}, \\
\Omega & \\
= & \frac{k_{\omega}\left(U_{, r}^{2}+U_{, z}^{2}\right)((1+\beta)+2 U /(1-U)) \pm \sqrt{\left(U_{, r}^{2}+U_{, z}^{2}\right) D}}{2(1+\beta) r(1-U)^{2} U_{, r}-2\left(U_{, r}^{2}+U_{, z}^{2}\right) A}, \\
D & 4(1+\beta) r(1-U) U_{, r} \\
& +\left(U_{, r}^{2}+U_{, z}^{2}\right)\left(k_{\omega}^{2}(1+\beta)^{2}-4 \beta r^{2}\right), \\
A= & \beta r^{2}(1-U)+k_{\omega}^{2} U\left(1+\beta+\frac{U}{1-U}\right),
\end{array}
$$

respectively. All the quantities depend on $r$ and $z$. In Figures 3(a) and 4(a) we show the behavior of the velocities $v_{0}^{2}$ and $v_{1}^{2}$ of a charged particle following "magnetogeodesic" motion on the halo for the values of indicated parameters, respectively. Additionally, in Figures 3(b) and 4(b), we plot the $z$-slices of the surface plot of the velocity and $v_{0}^{2}$ and $v_{1}^{2}$ for the indicated values of the parameters, respectively. These curves are obtained via vertical slices of the surface $v^{2}=$ $v^{2}(r, z)$ (a vertical slice is a curve formed by the intersection of the surface $v^{2}=v^{2}(r, z)$ with the vertical planes). For each curve, we can see that the velocity is always less than 1 , its maximum occurs around $r=0$, and it vanishes sufficiently fast as $r$ increases. It can also be observed that the maximum of the velocity decreases as the values of $z$ increase. We also computed these functions for other values of the parameters within the allowed range and in all cases we found a similar behavior. Naturally, the description of the motion of charged particles on disk here deduced is in agreement with the results of analysis of the magnetogeodesic motion of the particle in the magnetized disks discussed in [59].

\section{Conclusions}

We used the formalism presented in [54] to model an exact relativistic rotating disk surrounded by a magnetized halo. The model was obtained by solving the Einstein-Maxwell distributional field equations. In doing so, we introduced an auxiliary harmonic function that determines the functional dependence of the metric components and the electromagnetic potential. Accordingly, we separated the total energymomentum tensor of the system disk-halo. Additionally, we expressed the energy-momentum tensor of the halo as a sum of two distributional contributions, one due to the electromagnetic part and the other due to a material part. As we can see, because the spacetime here considered is nonstatic (conformastationary), the distributional approach of the Einstein-Maxwell equations allows us to work with a strongly nonlineal partial equations system. We considered, for simplicity, the astrophysical consistent case in that there is no electric charge on the halo. We obtained that the charge density on the disk is zero. 


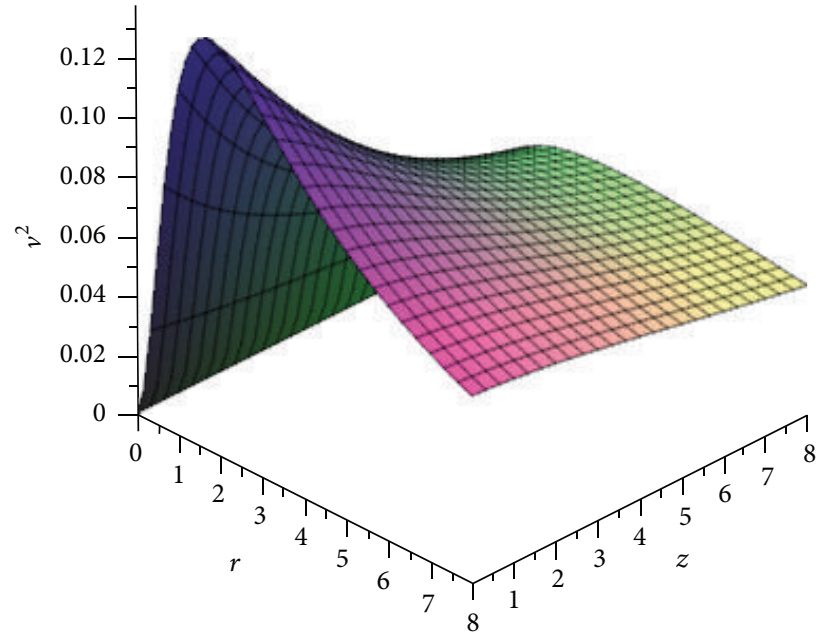

(a)

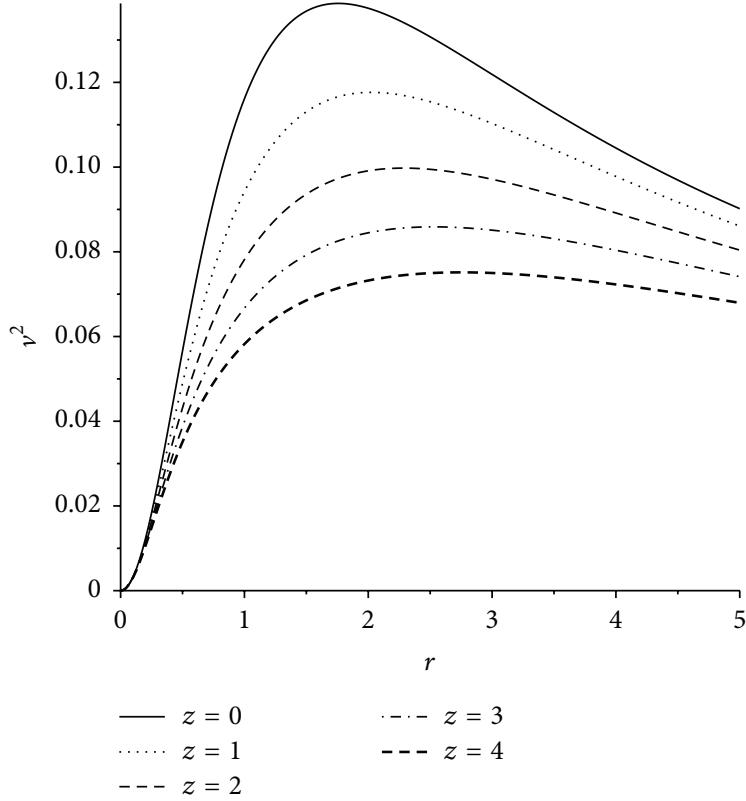

(b)

FIGURE 3: Surface plot of the velocity (a) $v_{0}^{2}$ and $z$-slices of the surface plot of the velocity (b) on the halo as functions depending on $r$ and $z$ with parameters $a=b_{0}=b_{1}=k=k_{\omega}=1$ and $\beta=0.75$.

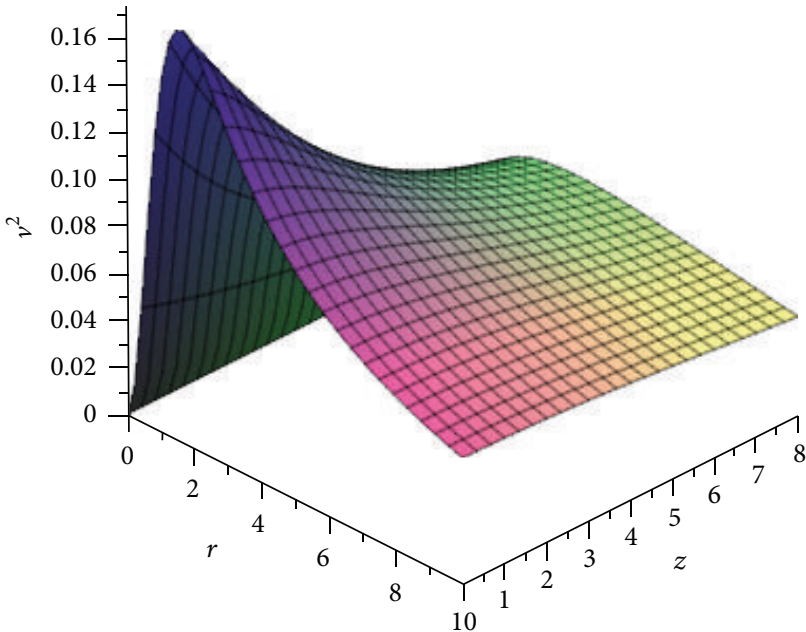

(a)

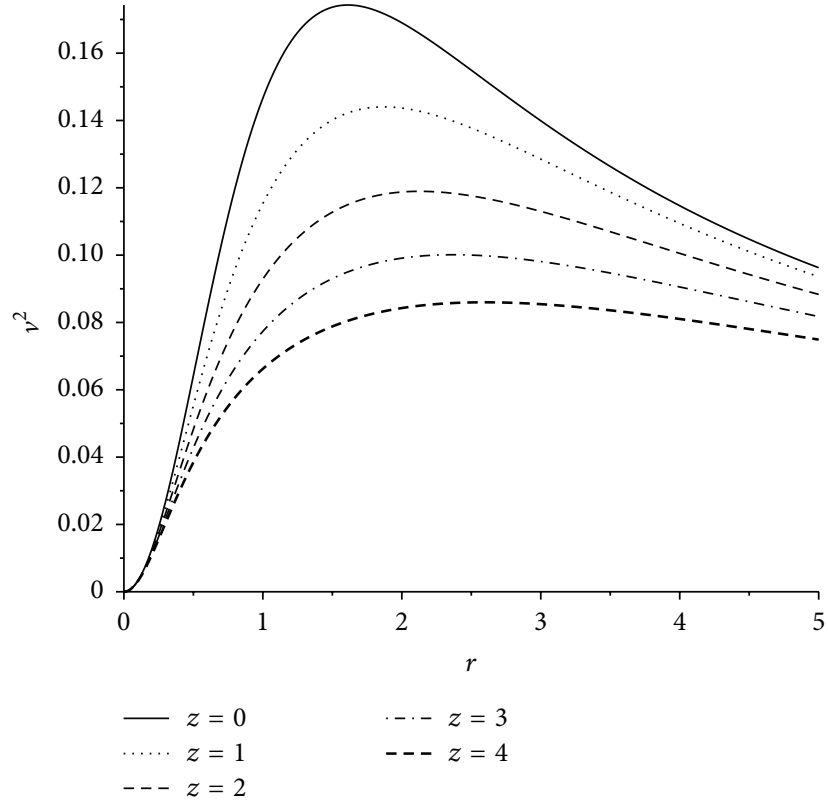

(b)

FIGURE 4: Surface plot of the velocity (a) $v_{1}^{2}$ and $z$-slices of the surface plot of the velocity (b) on the halo as functions depending on $r$ and $z$ with parameters $a=b_{0}=b_{1}=k=k_{\omega}=1$ and $\beta=0.75$.

In order to analyze the physical content of the energymomentum tensor of the halo and disk, we projected each tensor, in the canonical form, in the comoving frame defined by the local observers tetrad. This analysis allowed us to give a complete dynamical description of the system in terms of two parameters (i.e., $\beta$ and $k_{\omega}$ ) which determine the matter content of the sources. Indeed, the parameter $\beta$ in the metric vanishes when it is equal to the isotropic pressure and the anisotropic tensor on the material constituting the disk. Similarly, when the parameter $k_{\omega}$ is equal to zero the heat flux on the disk and halo vanishes, a feature of the static systems. So, in this paper we presented, for first 
time, the complete analysis of the most general energymomentum tensor of a disk-haloes system obtained from exact conformastationary axially symmetric solutions of the Einstein-Maxwell equations.

The expressions obtained here are the generalization of the expressions obtained for the conformastatic diskhaloes without isotropic pressure, stress tensor, or heat flow presented in [54]. Moreover, when we take simultaneously $k_{\omega}=0$ and $\beta=1$, we obtain the corresponding electrized disk-haloes version. Furthermore, our results are compatible with the description of the relativistic models of perfect fluid disks in a magnetic field presented in [59] and the halo presented in [53]. Furthermore, we have shown that the description of the motion of charged particles on the disk is in agreement with the results of analysis of particles motion in the magnetized disks discussed in [59]. In accordance with the results presented in $[53,65]$, it is also worth noticing that one can fix the values of the parameters $\beta, k_{\omega}$, and $b_{n}$ as well as the number of members of the particular solutions presented here in order to have velocity increasing linearly with radius of the disk.

We have considered specific solutions in which the gravitational and magnetic potential are completely determined by a "generalization" of the Kuzmin-disk potential. Accordingly, we have generated relativistic exact solutions for magnetized haloes surrounding rotating disks from a Newtonian gravitational potential of a static axisymmetric distribution of matter. The solution obtained is asymptotically Minkowskian in general and turns out to be free of singularities.

In short, we concluded that we have presented a wellbehaved exact general relativistic rotating disk surrounded by a well-behaved magnetized "material" halo. In our description we do not impose restriction on the kind of "material" constituting the system disk-halo. Consequently, we can speculate that the halo could be made of magnetized dark matter. This work provides a solid footing to refine future studies of relativistic disk-haloes systems and applications, for example, relativistic generalization of alpha-effect which will be discussed somewhere.

\section{Appendix}

\section{The Local Observers}

We write metric (7) in the form

$$
d s^{2}=-F(d t+\omega d \varphi)^{2}+F^{-\beta}\left[d r^{2}+d z^{2}+r^{2} d \varphi^{2}\right],
$$

where we have rewritten $F=e^{2 \phi}$. In addition, we define the tetrad of the local observers $h^{(\alpha)}{ }_{\mu}$, in which the metric has locally the form of Minkowskian metric

$$
d s^{2}=\eta_{(\mu)(\nu)} \mathbf{h}^{(\mu)} \otimes \mathbf{h}^{(\nu)}
$$

and is given by

$$
\begin{aligned}
& h_{\alpha}^{(t)}=F^{1 / 2}\{1,0,0, \omega\}, \\
& h_{\alpha}^{(r)}=F^{-\beta / 2}\{0,1,0,0\},
\end{aligned}
$$

$$
\begin{aligned}
& h_{\alpha}^{(z)}=F^{-\beta / 2}\{0,0,1,0\}, \\
& h_{\alpha}^{(\varphi)}=F^{-\beta / 2}\{0,0,0, r\} .
\end{aligned}
$$

The dual tetrad reads

$$
\begin{aligned}
& h_{(t)}{ }^{\alpha}=F^{-1 / 2}\{1,0,0,0\}, \\
& h_{(r)}{ }^{\alpha}=F^{\beta / 2}\{0,1,0,0\}, \\
& h_{(z)}{ }^{\alpha}=F^{\beta / 2}\{0,0,1,0\}, \\
& h_{(\varphi)}{ }^{\alpha}=\frac{F^{\beta / 2}}{r}\{-\omega, 0,0,1\} .
\end{aligned}
$$

The circular velocity of the system disk-halo can be modelled by a fluid spacetime whose circular velocity $V^{\alpha}$ can be written in terms of two Killing vectors $t^{\alpha}$ and $\varphi^{\alpha}$ :

$$
V^{\alpha}=V^{t}\left(t^{\alpha}+\Omega \varphi^{\alpha}\right)
$$

where

$$
\Omega \equiv \frac{u^{\varphi}}{u^{t}}=\frac{d \varphi}{d t}
$$

is the angular velocity of the fluid as seen by an observer at rest at infinity. The velocity satisfies the normalization $V_{\alpha} V^{\alpha}=-1$. Accordingly, for metric (A.1) we have

$$
\left(V^{t}\right)^{2}=\frac{1}{-t^{\alpha} t_{\alpha}-2 \Omega t^{\alpha} \varphi_{\alpha}-\Omega \varphi^{\alpha} \varphi_{\alpha}}
$$

with

$$
\begin{aligned}
t^{\alpha} t_{\alpha} & =g_{t t}=-F, \\
t^{\alpha} \varphi_{\alpha} & =g_{t \varphi}=-\omega F, \\
\varphi^{\alpha} \varphi_{\alpha} & =g_{\varphi \varphi}=r^{2} F^{-\beta}\left(1-F^{1+\beta} \frac{\omega^{2}}{r^{2}}\right),
\end{aligned}
$$

and consequently we write the velocity as

$$
V^{t}=\frac{1}{F^{1 / 2}(1+\omega \Omega) \sqrt{1-V_{\mathrm{LOC}}^{2}}},
$$

where

$$
V_{\mathrm{LOC}} \equiv \frac{r \Omega}{F^{(1+\beta) / 2}(1+\omega \Omega)}
$$

is the velocity as measured by the local observers.

\section{Conflict of Interests}

The authors declare that there is no conflict of interests regarding the publication of this paper. 


\section{References}

[1] M. Krause, "Magnetic fields and halos inspiral galaxies," http://arxiv.org/abs/1401.1317.

[2] M. Krause, "Magnetic fields in spiral galaxies. Highlights of Astronomy," Proceedings of the International Astronomical Union, vol. 10, p. 399, 2012.

[3] D. Ryu, D. R. G. Schleicher, R. A. Treumann, C. G. Tsagas, and L. M. Widrow, "Magnetic fields in the large-scale structure of the universe," Space Science Reviews, vol. 166, no. 1-4, pp. 1-35, 2012.

[4] J. Han, "Magnetic fields in our Milky Way Galaxy and nearby galaxies," Proceedings of the International Astronomical Union, vol. 8, pp. 213-224, 2012.

[5] W. B. Bonnor and A. Sackfield, "The interpretation of some spheroidal metrics," Communications in Mathematical Physics, vol. 8, no. 4, pp. 338-344, 1968.

[6] T. Morgan and L. Morgan, "The gravitational field of a disk," Physical Review, vol. 183, no. 5, article 1097, 1969.

[7] L. Morgan and T. Morgan, "Gravitational field of shells and disks in general relativity," Physical Review D, vol. 2, no. 12, article 2756, 1970.

[8] D. Lynden-Bell, S. Pineault, and R. Astron, "Relativistic disksI. Counter rotating disks," Monthly Notices of the Royal Astronomical Society, vol. 185, no. 4, pp. 679-694, 1978.

[9] B. H. Voorhees, "Relativistic disks. I. Background models," Physical Review D, vol. 5, no. 10, pp. 2413-2418, 1972.

[10] A. Chamorro, R. Gregory, and J. M. Stewart, "Static axisymmetric discs and gravitational collapse," The Royal Society of London-Series A: Proceedings, vol. 413, no. 1844, pp. 251-262, 1987.

[11] P. S. Letelier and S. R. Oliveira, "Exact self-gravitating disks and rings: a solitonic approach," Journal of Mathematical Physics, vol. 28, no. 1, pp. 165-170, 1987.

[12] J. P. S. Lemos, "Self-similar relativistic discs with pressure," Classical and Quantum Gravity, vol. 6, no. 9, pp. 1219-1230, 1989.

[13] J. Bičák, D. Lynden-Bell, and J. Katz, "Relativistic disks as sources of static vacuum spacetimes," Physical Review D, vol. 47, no. 10 , pp. 4334-4343, 1993.

[14] J. Bičák, D. Lynden-Bell, and C. Pichon, "Relativistic discs and flat galaxy models," Monthly Notices of the Royal Astronomical Society, vol. 265, no. 1, pp. 126-144, 1993.

[15] G. A. González and P. S. Letelier, "Relativistic static thin discs with radial stress support," Classical and Quantum Gravity, vol. 16, no. 2, pp. 479-494, 1999.

[16] G. A. González and O. A. Espitia, "Relativistic static thin disks: the counterrotating model," Physical Review. D, vol. 68, no. 10, Article ID 104028, 2003.

[17] G. A. González, A. C. Gutiérrez-Piñeres, and V. M. ViñaCervantes, "Relativistic static thin dust disks with an inner edge: an infinite family of new exact solutions," Physical Review D, vol. 79, no. 12, Article ID 124048, 2009.

[18] D. Lynden-Bell and S. Pineault, "Relativistic disks-II. Selfsimilar disks in rotation," Monthly Notices of the Royal Astronomical Society, vol. 185, no. 4, pp. 695-712, 1978.

[19] J. Bičák and T. Ledvinka, "Relativistic disks as sources of the Kerr metric," Physical Review Letters, vol. 71, no. 11, pp. 16691672, 1993.

[20] C. Pichon and D. Lynden-Bell, "New sources for Kerr and other metrics: rotating relativistic discs with pressure support," Monthly Notices of the Royal Astronomical Society, vol. 280, pp. 1007-1026, 1996.
[21] G. A. González and P. S. Letelier, "Rotating relativistic thin disks," Physical Review D, vol. 62, no. 6, Article ID 064025, 8 pages, 2000.

[22] G. A. González and A. C. Gutiérrez-Piñeres, "Stationary axially symmetric relativistic thin discs with nonzero radial pressure," Classical and Quantum Gravity, vol. 29, no. 13, Article ID 135001, 2012.

[23] J. P. S. Lemos and P. S. Letelier, "Superposition of MORgan and MORgan discs with a Schwarzschild black hole," Classical and Quantum Gravity, vol. 10, no. 6, pp. L75-L78, 1993.

[24] J. P. S. Lemos and P. S. Letelier, "Exact general relativistic thin disks around black holes," Physical Review D, vol. 49, no. 10, pp. 5135-5143, 1994.

[25] J. P. Lemos and P. S. Letelier, "Two families of exact disks with a central black hole," International Journal of Modern Physics D: Gravitation, Astrophysics, Cosmology, vol. 5, no. 1, pp. 53-63, 1996.

[26] O. Semerák and M. Zácek, "Gravitating discs around a Schwarzschild black hole: I," Classical and Quantum Gravity, vol. 17, no. 7, pp. 1613-1626, 2000.

[27] O. Semerák, "Thin disc around a rotating black hole, but with support in-between," Classical and Quantum Gravity, vol. 19, no. 14, pp. 3829-3844, 2002.

[28] M. Žáček and O. Semerák, "Gravitating discs around a Schwarzschild black hole II," Czechoslovak Journal of Physics, vol. 52, no. 1, pp. 19-27, 2002.

[29] O. Semerák, "Gravitating discs around a Schwarzschild black hole. III," Classical and Quantum Gravity, vol. 20, no. 9, pp. 16131634, 2003.

[30] O. Semerák, "Exact power-law discs around static black holes," Classical and Quantum Gravity, vol. 21, no. 8, pp. 2203-2218, 2004.

[31] V. Karas, J. Huré, and O. Semerák, "Gravitating discs around black holes," Classical and Quantum Gravity, vol. 21, no. 7, 2004.

[32] A. C. Gutiérrez-Piñeres, G. García-Reyes, and G. A. González, "Exact relativistic models of thin disks around static black holes in a magnetic field," International Journal of Modern Physics D, vol. 23, no. 1, Article ID 1450010, 23 pages, 2014.

[33] A. Feinstein, J. Ibañez, and R. Lazkoz, "Disks in expanding FRW universes," The Astrophysical Journal, vol. 495, no. 1, p. 131, 1998.

[34] D. Vogt and P. S. Letelier, "Exact general relativistic perfect fluid disks with halos," Physical Review D, vol. 68, Article ID 084010, 2003.

[35] M. Ujevic and P. S. Letelier, "Stability of general relativistic geometric thin disks," Physical Review D, vol. 70, no. 8, Article ID 084015, 2004.

[36] T. Ledvinka, M. Žofka, and J. Bičák, "Relativistic disks as sources of Kerr-Newman fields," in Recent Developments in Theoretical and Experimental General Relativity, Gravitation, and Relativistic Field Theories, T. Piran and R. Ruffini, Eds., p. 339, 1999.

[37] G. García-Reyes and G. A. González, "Rotating and counterrotating relativistic thin disks as sources of stationary electrovacuum spacetimes," Brazilian Journal of Physics, vol. 37, no. 3, pp. 1094-1105, 2007.

[38] P. S. Letelier, "Exact general relativistic disks with magnetic fields," Physical Review D, vol. 60, no. 10, Article ID 104042, 1999.

[39] A. C. Gutiérrez-Piñeres and G. A. González, "An infinite family of magnetized morgan-morgan relativistic thin disks," International Journal of Theoretical Physics, vol. 51, no. 6, pp. 1737-1752, 2012. 
[40] D. Vogt and P. S. Letelier, "Exact relativistic static charged dust discs and non-axisymmetric structures," Classical and Quantum Gravity, vol. 21, no. 14, pp. 3369-3378, 2004.

[41] J. Katz, J. Bicák, and D. Lynden-Bell, "Disc sources for conformastationary metrics," Classical and Quantum Gravity, vol. 16, no. 12, pp. 4023-4034, 1999.

[42] G. A. González, A. C. Gutiérrez-Piñeres, and P. A. Ospina, "Finite axisymmetric charged dust disks in conformastatic spacetimes," Physical Review D, vol. 78, no. 6, Article ID 064058, 2008.

[43] R. G. García and G. A. González, "Electrovacuum static counterrotating relativistic dust disks," Physical Review D, vol. 69, no. 12, Article ID 124002, 2004.

[44] D. Vogt and P. S. Letelier, "Exact relativistic static charged perfect fluid disks," Physical Review D, vol. 70, no. 6, Article ID 064003, 6 pages, 2004.

[45] G. García-Reyes and G. A. González, "Counterrotating perfect fluid discs as sources of electrovacuum static spacetimes," Classical and Quantum Gravity, vol. 21, no. 21, article 4845, 2004.

[46] G. García-Reyes and G. A. González, "Charged perfect fluid disks as sources of Taub-NUT-type spacetimes," Physical Review D, vol. 70, no. 10, Article ID 104005, 2004.

[47] N. Gürlebeck, J. Bičák, and A. C. Gutiérrez-Piñeres, "Monopole and dipole layers in curved spacetimes: formalism and examples," Physical Review D, vol. 83, no. 12, Article ID 124023, 2011.

[48] N. Gürlebeck, J. Bičák, and A. C. Gutiérrez-Piñeres, "Electromagnetic sources distributed on shells in a Schwarzschild background," General Relativity and Gravitation, vol. 43, no. 12, pp. 3301-3312, 2011.

[49] M. Zamaninasab, E. Clausen-Brown, T. Savolainen, and A. Tchekhovskoy, "Dynamically important magnetic fields near accreting supermassive black holes," Nature, vol. 510, no. 7503, pp. 126-128, 2014.

[50] R. Beck, "Magnetic fields in galaxies," in Magnetic Fields in Diffuse Media, vol. 407 of Astrophysics and Space Science Library, pp. 507-528, Springer, Berlin, Germany, 2015.

[51] F. De Paolis, V. Gurzadyan, A. Nucita et al., "Planck confirmation of the disk and halo rotation of M 31," Astronomy and Astrophysics, vol. 565, article L3, 2014.

[52] E. Mikhailov, A. Kasparova, D. Moss, R. Beck, D. Sokoloff, and A. Zasov, "Magnetic fields near the peripheries of galactic discs," Astronomy \& Astrophysics, vol. 568, article A66, 2014.

[53] K. Chakraborty, F. Rahaman, S. Ray, A. Nandi, and N. Islam, "Possible features of galactic halo with electric field and observational constraints," General Relativity and Gravitation, vol. 46, no. 10, article 1807, 2014.

[54] A. C. Gutiérrez-Piñeres, G. A. González, and H. Quevedo, "Conformastatic disk-haloes in Einstein-Maxwell gravity," Physical Review D, vol. 87, no. 4, Article ID 044010, 2013.

[55] A. C. Gutiérrez-Piñeres, C. S. Lopez-Monsalvo, and H. Quevedo, "Variational thermodynamics of relativistic thin disks," General Relativity and Gravitation, vol. 47, article 144, 2013.

[56] A. C. Gutiérrez-Piñeres, "Conformastationary disk-haloes in Einstein-Maxwell gravity," General Relativity and Gravitation, vol. 47, no. 5, article 54, 2015.

[57] H. Stephani, D. Kramer, M. MacCallum, C. Hoenselaers, and E. Herlt, Exact Solutions of Einstein's Field Equations, Cambridge University Press, 2003.
[58] J. Synge, Relativity: The General Theory, North-Holland, Interscience, Amsterdam, The Netherlands, 1960.

[59] G. García-Reyes and O. A. Espitia, "Exact relativistic models of perfect fluid disks in a magnetic field," General Relativity and Gravitation, vol. 46, article 1674, 2014.

[60] P. S. Letelier and A. Wang, "Space-time defects," Journal of Mathematical Physics, vol. 36, no. 6, pp. 3023-3042, 1995.

[61] J. Binney and S. Tremaine, Princeton Series in Astrophysics, Princeton University Press, Princeton, NJ, USA, 1998.

[62] G. Arfken, Mathematical Methods for Physicists, vol. 1, Academic Press, New York, NY, USA, 1966.

[63] M. Carmeli, Classical Fields, John Wiley \& Sons, 1982.

[64] L. D. Landau and E. M. Lifshits, The Classical Theory of Fields, vol. 2, Butterworth-Heinemann, 1975.

[65] K. K. Nandi, A. I. Filippov, F. Rahaman et al., "Features of galactic halo in a brane world model and observational constraints," Monthly Notices of the Royal Astronomical Society, vol. 399, no. 4, pp. 2079-2087, 2009. 


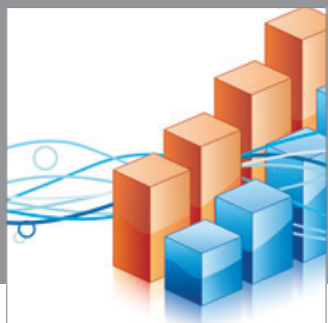

Advances in

Operations Research

mansans

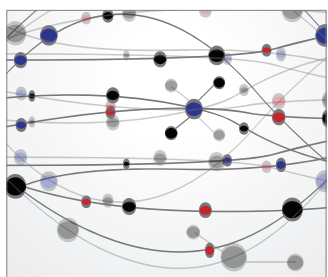

The Scientific World Journal
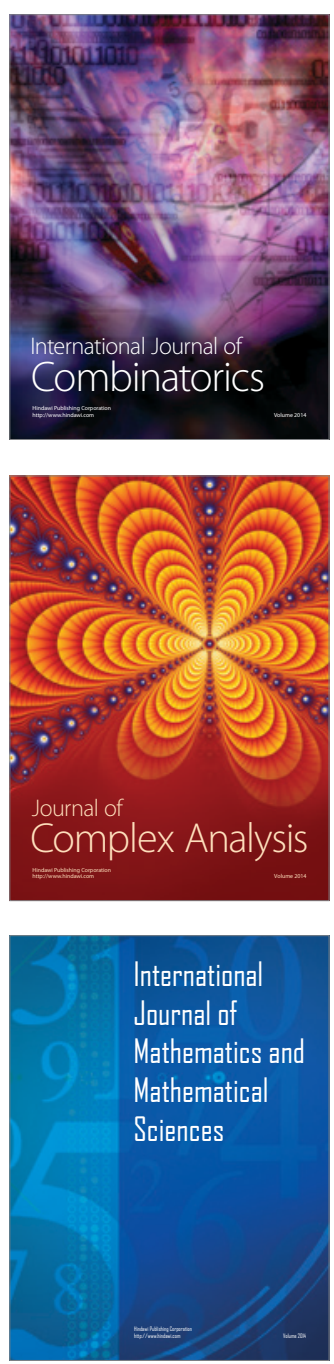
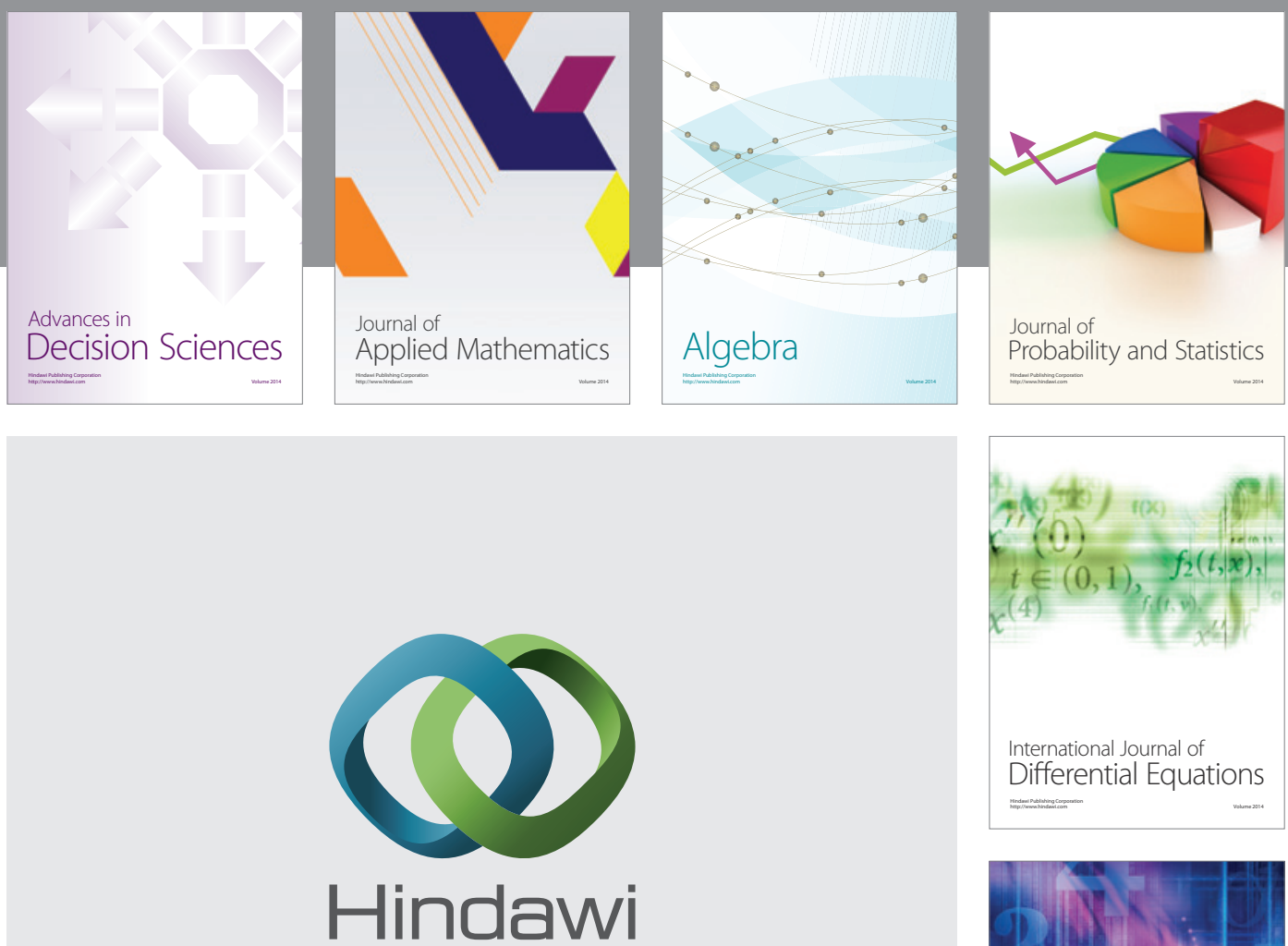

Submit your manuscripts at http://www.hindawi.com
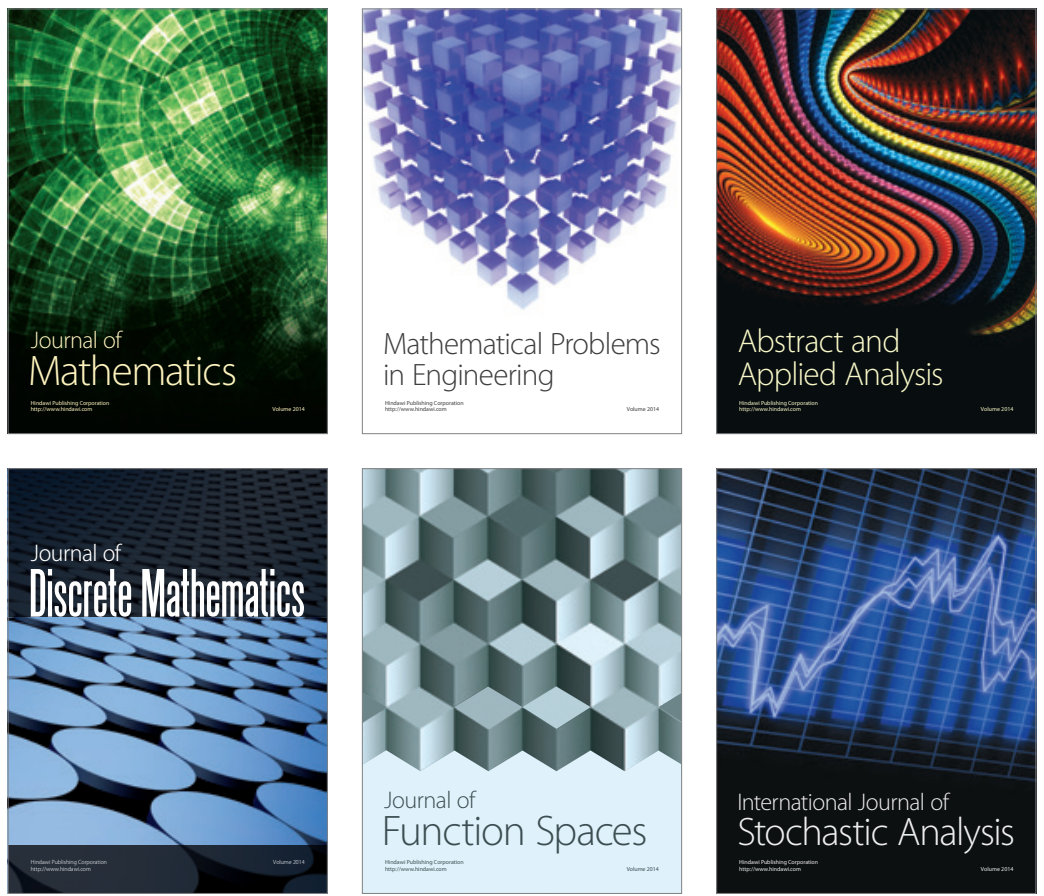

Journal of

Function Spaces

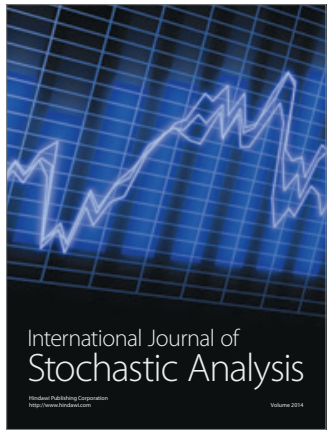

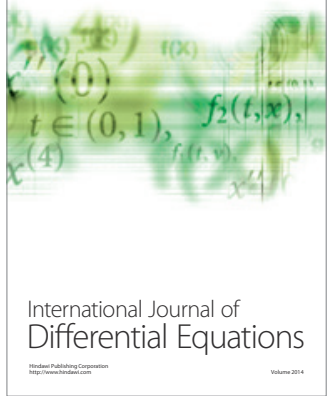
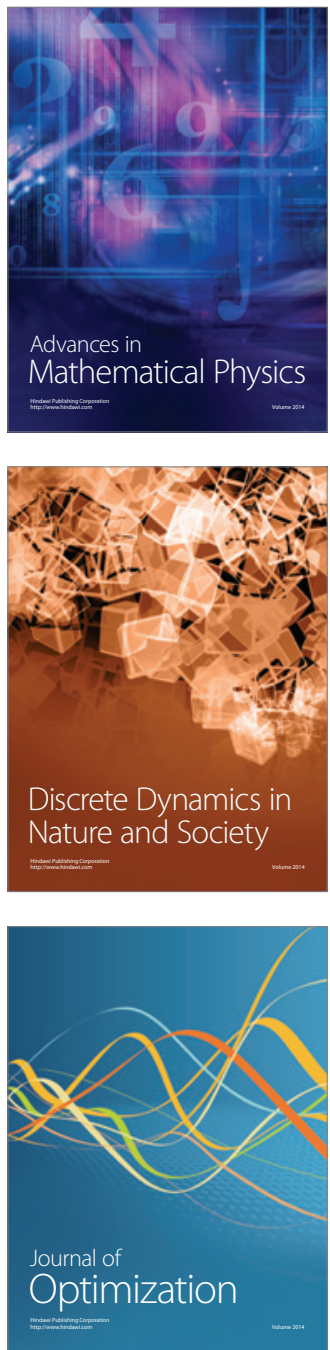